\title{
Modification and Machining on Back Surface of a Silicon Substrate by Femtosecond Laser Pulses at 1552 nm
}

\author{
Yoshiro ITO*, Hiroki SAKASHITA, Ryosuke SUZUKI, Mitsuru UEWADA, Khanh Phu LUONG and Rie TANABE \\ Department of Mechanical Engineering, Nagaoka University of Technology, \\ 1603-1 Kamitomioka, Nagaoka, Niigata 940-2188, Japan \\ E-mail: itoy@vos.nagaokaut.ac.jp
}

\begin{abstract}
Silicon ( $\mathrm{Si}$ ) is widely used material in microelectronics, MEMS, photonics and more. Although $\mathrm{Si}$ is not transparent for commonly used processing lasers in near infrared to ultraviolet spectral range, the light at low energy than its band gap energy should be transmit through Si substrate. Using a short pulse laser in this wavelength range, non-linear machining of Si should be performed just like non-linear processing of transparent materials by conventional lasers. In this study, we have been studied a laser grooving on back surface of Si substrate by an infrared femtosecond laser. The laser was focused at the back of the Si substrate. The top surface of the Si showed no change while morphology of back surface changes into aggregation of small particles, the size of which is about $500 \mathrm{~nm}$. From cross sectional view of the Si substrate, a structural change was observed in regions close to the focus position. Raman spectra indicate that the single crystal Si was transformed into amorphous and poly-crystal Si in irradiated region. Although machining for the back surface of the $\mathrm{Si}$ is quite difficult, machining of the grooves of few micrometers was achieved in the laser-assisted wet etching using $\mathrm{KOH}$ solution.

DOI: $10.2961 /$ jlmn.2014.02.0004
\end{abstract}

Keywords: silicon, back surface, modification, near-infrared, femtosecond laser

\section{Introduction}

Processing of transparent materials via non-linear absorption mechanisms induced by short pulse lasers is powerful technology to modify or machine inside and/or backside of materials in three dimensional way $[1,2]$. This is a unique method which only laser processing can achieve and has been applied in many fields. Single crystal silicon (c-Si) is widely used material in microelectronics, MEMS, photonics and many other fields. $\mathrm{Si}$ is, however, not transparent for commonly used processing lasers in near infrared to ultraviolet spectral range and has not been a subject for the non-linear processing by commonly used lasers so far.

It is suggested that when weakly absorbed visible or near infrared nanosecond laser pulses are focused inside of $\mathrm{Si}$ substrate, the laser could induce small cracks inside $\mathrm{Si}$, which make possible to dice the substrate [3, 4]. The proposed mechanism for this process induced by nanosecond visible laser is that initial temperature rise around the focus point due to small linear absorption causes the increase of absorption coefficient, thus a positive feedback process starts so that enough energy is deposited to form micro-cracks [5-7]. Attempts for processing inside Si by femtosecond laser pulses at $800 \mathrm{~nm}$ were reported by T. Chen et. al. [8] and C. Li et. al. [9,10]. Some voids were formed interior of $\mathrm{Si}$ substrates, just below the front surface, with some structural changes. Laser radiation at this wavelength is, however, absorbed by $\mathrm{Si}$ and thus machining processes are not flexible compared with machining of transparent materials.

A. H. Nejadmalayeri et al. reported on inscription of optical waveguides in c-Si using $2.4 \mu \mathrm{m}$ radiation of $70 \mathrm{fs}$ from an optical parametric amplifier [11]. In their report, however, inscription of optical waveguide in $\mathrm{c}-\mathrm{Si}$ was achieved only at 5-20 $\mu \mathrm{m}$ below the silica-Si interface.

We have demonstrated that $\mathrm{Si}$ can be treated as a transparent substrate like glass in infrared region indeed and that micromachining of substrates through a $\mathrm{Si}$ substrate by a femtosecond laser at $1552 \mathrm{~nm}$ is possible. A crystal oscillator frequency packaged inside a $\mathrm{Si}$ can be increased precisely by irradiating its surface with the laser from outside the package $[12,13]$.

We have extended our study to exploit possibility of 3D machining of $\mathrm{Si}$ by non-linear absorption just like 3D machining of ordinal transparent materials. We have studied the micromachining on the backside of the $\mathrm{Si}$ substrate by using a near-infrared $1552 \mathrm{~nm}$ femtosecond laser. From our previous research, it is found that precise focusing of the laser on the back surface of $\mathrm{Si}$ is a key to achieve modification and machining of the back surface $[14,15]$.

Recently, subsurface modification of Si by an infrared femtosecond laser at $1550 \mathrm{~nm}$ was reported and stripe structure of modified zone inside $\mathrm{Si}$ was formed. They showed that these stripe structures acted as a diffraction grating [16].

In this paper, attempts for surface modification and grooving on back surface of Si substrate are reported. To focus the infrared laser pulse at designated position, an infrared microscope was combined with laser irradiating system and the lens with a correction collar was used to compensate the aberration caused by large refractive index of Si. 


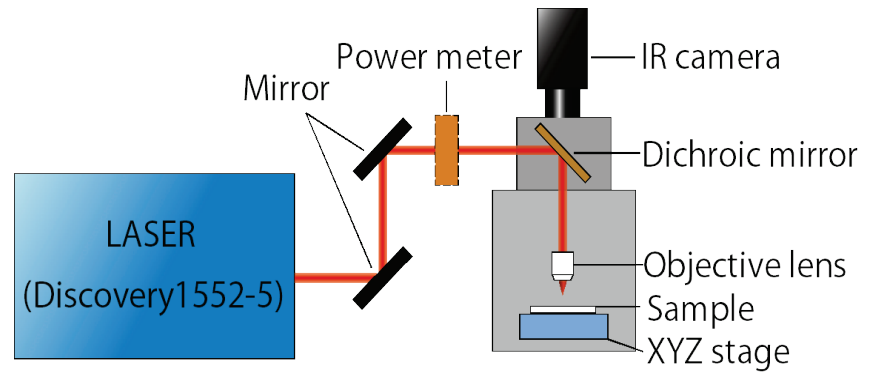

Fig.1 Schematic diagram of experimental setups.

\section{Experimental}

Figure 1 shows a schematic diagram of experimental set up. An ultrafast fiber laser, Raydiance Co. Discovery 1552-5, was used as a machining source. The laser emits 5 $\mu \mathrm{J}, 900 \mathrm{fs}$ pulses at $1552.5 \mathrm{~nm}$ with frequencies up to 500 $\mathrm{kHz}$. Pulse energy was adjusted to $4 \mu \mathrm{J}$ in front of microscope.

To observe an irradiated point during the laser irradiation, an infrared optical microscope was added in the machining system. The microscope was equipped with a dichroic mirror, which reflected the wavelength of $1552 \mathrm{~nm}$ and passed through that of $1100 \mathrm{~nm}$. The output from the laser was focused using an objective lens of x100 designed for IR light with NA 0.85 (Olympus LC Plan N 100x/0.85 IR). The lens is equipped with a correction collar for compensation of aberration induced by both glass and $\mathrm{Si}$, amount of which is indicated as thicknesses of both materials. As the minimum scale on the collar was equivalent to the Si thickness of $100 \mu \mathrm{m}$, we interpolated the scale linearly down to $25 \mu \mathrm{m}$. However, the wavelength used for determining these scale was not provided from the manufacturer. To determine required correction, a small dot mark was made on the surface of the Si substrate by irradiating focused laser pulses directly, the substrate was turned upside-down and the mark was observed through the microscope at $1100 \mathrm{~nm}$ from opposite side (hereafter 'front side') and the collar position was adjusted to get clear image of the mark. Cover glass of $0.15 \mathrm{~mm}$ thick was placed after the objective lens to protect the lens. Therefore, the experimentally determined position of the collar was expected to compensate the thickness of both Si substrate and cover glass, and was $0.575 \mathrm{~mm}$ of Si thickness scale. This value should be considered as a relative indicator of the amount of correction made by the collar and we will use only the numerical values hereafter to indicate correction collar positions.

A spot size measured at this focus point was estimated to be $3 \mu \mathrm{m}$ and the laser was operated at the repetition rate of $100 \mathrm{kHz}$. A p-type Si substrate of $320 \mu \mathrm{m}$ thickness was used.

Irradiated samples were observed by an optical microscope at both infrared $(1100 \mathrm{~nm})$ and visible (with an infrared cut filter), as well as a scanning electron microscope (SEM). A micro laser-Raman spectrometer was used to analyze the structure of machined area.

\section{Results and discussion}

\subsection{Effects of focus position}

Figure 2 shows visible and infrared (IR) microscope images of back surface of machined Si substrate observed from the backside. The position of the collar was set at 0.575 as described before. Focus position of $\pm 0 \mu \mathrm{m}$ was determined in this position of the collar. Because the resolution of the collar was not accurate enough to focus the laser beam at the back surface, the laser was scanned in $500 \mu \mathrm{m}$ lines and $50 \mu \mathrm{m}$ space with changing the focus position, i.e., distance between the lens and the sample surface, stepwise by $1 \mu \mathrm{m}$ relative to the position of $\pm 0 \mu \mathrm{m}$. During these scans, the collar position was fixed at 0.575 . We expect that, even though this process introduces additional uncertainty in compensation of aberration by the collar, slight change of sample position could adjust the focus more precise than the collar resolution. Top image is obtained by observation in visible and bottom by observation in IR. In the focus position from $-12 \mu \mathrm{m}$ to 0 $\mu \mathrm{m}$, no change was observed in visible observation. On the other hand, gray lines were observed at all positions in the IR observation. These gray lines might be the same structural change reported by Sreenivas et. al even though they just suggested that the nature of the change would be a change in refractive index at that part [16]. Raman study on this line will be described in section 3.3.

Figure 3 shows the SEM image of the machined line that observed as black line at minus focus position in Fig. 2. Fine particle-like structure is observed and the diameter of this particle is about $500 \mathrm{~nm}$. It was also found that the surface on the gray line shown in figure 2 did not show any change detected from the SEM observation. This suggests that some changes in optical properties should occur only in inside of the substrate at the positions where only the gray lines were observed. The SEM observation of black lines appeared at positive focus positions shows the same microstructures appeared in Fig. 3. The SEM of front surface of the substrate facing to the laser showed no observable change at locations corresponding to these lines. Thus the position selective modification of the back surface

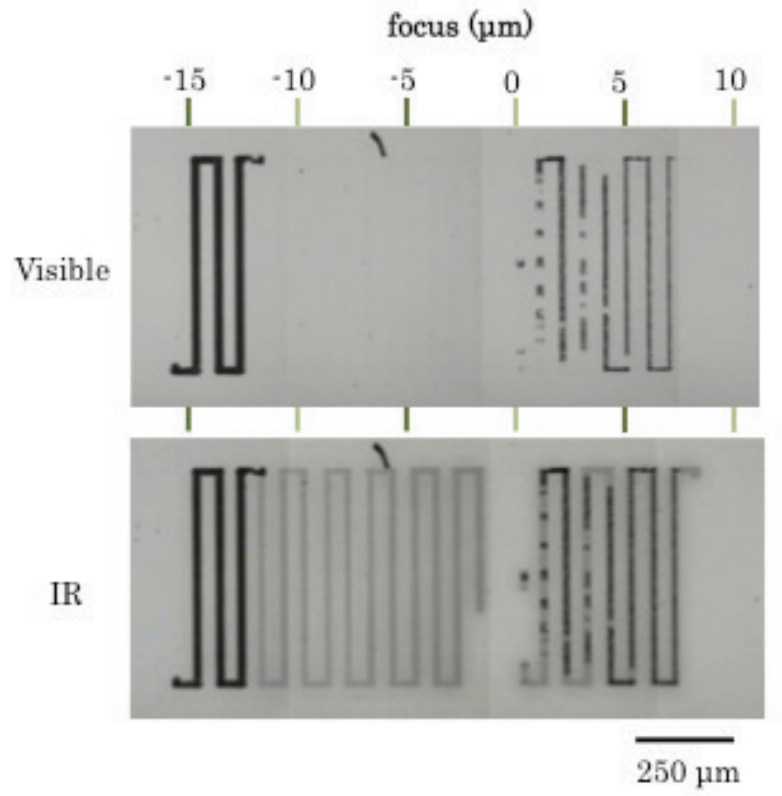

Fig. 2 Microscope images of back surface of machined Si substrate. Top image was obtained by visible observation and bottom was by IR observation. 


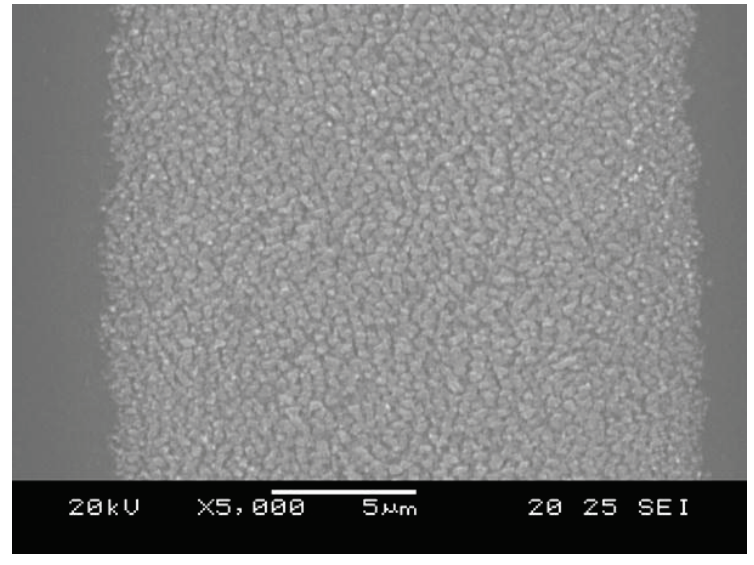

Fig.3 SEM image on the machined line of the back surface observed for focus position at $-15 \mu \mathrm{m}$.

of the Si substrate through itself is achieved.

There is a gap in the focus positions where the back surface was machined. Black lines are observed at -15 to -13 and at 2 to $7 \mu \mathrm{m}$ and only fractional changes are observed at $0 \mu \mathrm{m}$. The line width in minus positions is wider than that in plus positions. We considered that this might due to insufficient correction of aberration: The wavelength of the laser for machining is longer than that of the light for IR observation and the refractive index of $\mathrm{Si}$ differs at these two wavelengths, which results in insufficient correction at $1552 \mathrm{~nm}$. Therefore, the effect of the correction collar position was investigated to compensate this probable aberration. Additional correction of the collar should be needed to achieve precise irradiation at the back surface of the substrate, due to the difference of the refractive indices at $1100 \mathrm{~nm}$ and $1552 \mathrm{~nm}$.

\subsection{Effect of refractive index difference of $\mathrm{Si}$ at 1552 and $1100 \mathrm{~nm}$ : Additional correction}

To achieve required correction due to the difference of refractive index of $\mathrm{Si}$ at these two wavelengths, the procedure in previous section was repeated by changing the collar position stepwise. When the correction collar position was changed from 0.575 to 0.600 , processing lines of Si surface were observed at focus positions within \pm 2 $\mu \mathrm{m}$. We postulate that this correction value was the most plausible value under our current irradiation system.

Figure 4 shows visible (left) and IR (right) microscope images of the lines appeared at the back surface of Si after laser irradiation with collar position fixed at 0.600. Some black lines are observed at -2 to $+2 \mu \mathrm{m}$ in the visible observation with narrowest width at $+2 \mu \mathrm{m}$. Continuous gray lines overlapping with the black lines are also observed in the IR observation. Their width is broader compared with the black ones and the width is nearly the same at all positions. The gray line suggests that structure of inside the substrate has changed by laser irradiation and indicate possibility of inner structural modification and inside marking of $\mathrm{Si}$.

To study the changes occurred inside the Si substrate, it was cut perpendicular to the machined lines and its cross section was observed. The cross-section of as-cut samples showed no observable change. Therefore, the samples were etched by $\mathrm{KOH}$ solution at $30^{\circ} \mathrm{C}$ for $25 \mathrm{~min}$.

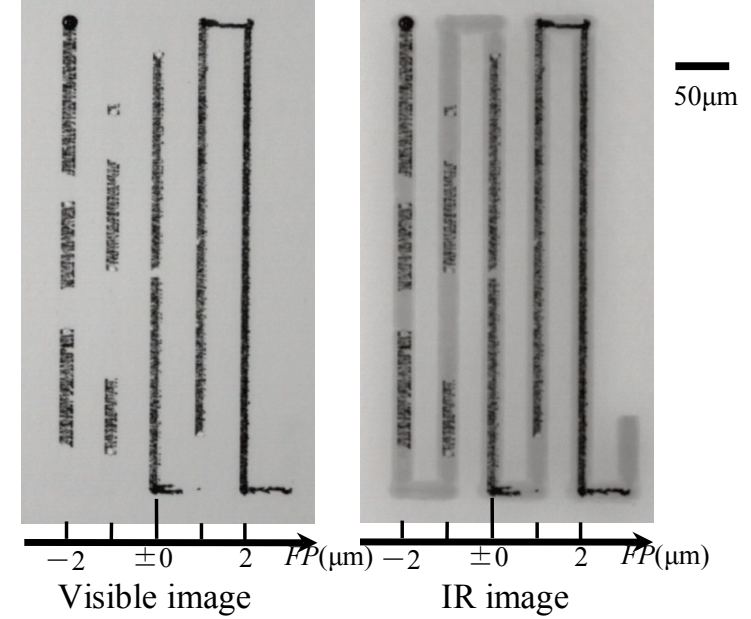

Fig.4 Visible (left) and IR (right) microscope images observed from back side obtained at collar position 0.600 .

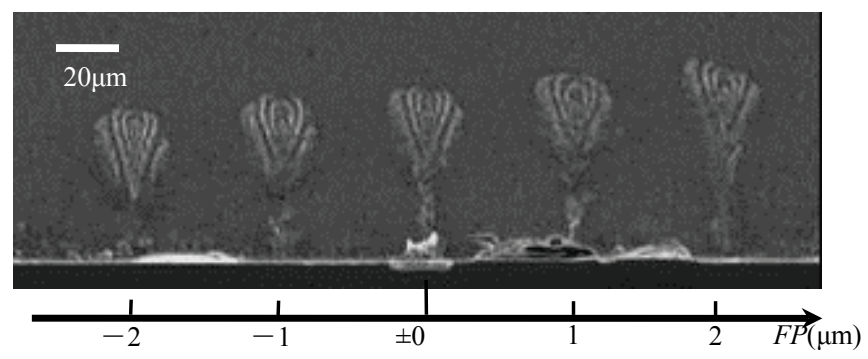

Fig. 5 Cross-sectional SEM image of gray lines after etching by $\mathrm{KOH}$ solution. Focus position is indicated as in Fig. 4. The line at the bottom is the back surface of the substrate.

Figure 5 shows cross-sectional SEM image of lines obtained at focus positions from -2 to $+2 \mu \mathrm{m}$. Changed regions appeared in white and in inversed triangle shape, which was narrow at the surface and wider inside of the $\mathrm{Si}$, were observed at positions corresponding to the gray lines. The whitened parts form a layered pattern looks like a bud of a flower cut in the center. Length of the changed region extends approximately 50 to $70 \mu \mathrm{m}$ inward from the back surface along with the movement of the focus towards inside. The largest width of the cross-sectional change coincides with the width of the gray line in IR observation. These results suggest that the gray area in IR image should correspond to some changes of Si structure which causes the change of etch rate by $\mathrm{KOH}$ solution as well.

\subsection{Analysis by laser Raman spectroscopy}

To examine the change on the laser irradiation, analysis by micro-Raman spectroscopy was applied. Figure 6 shows the Raman spectra measured at (a) not machined position and (b) machined position on the rare surface. In the position where laser was not irradiated, a peak at $525 \mathrm{~cm}^{-1}$ is observed as shown in Fig. 6 (a) and this line is known as Raman-shift of single-crystal Si [16]. On the other hand, the Raman spectra obtained at the position where the aggregation of small particles was formed by laser irradiation consists of a peak at $525 \mathrm{~cm}^{-1}$ and an additional 

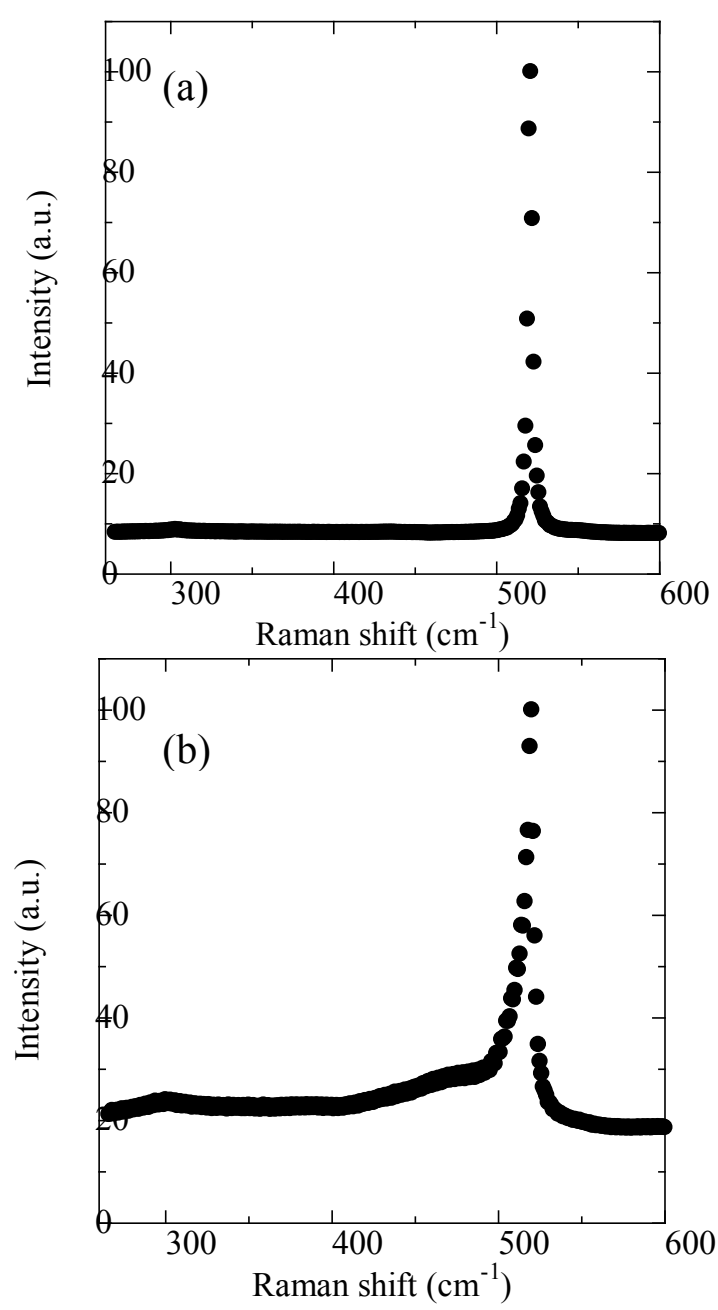

Fig. 6 Micro-Raman spectra of Si rear surface obtained at (a) not machined position and (b) machined position.

broad peak at $480 \mathrm{~cm}^{-1}$ as shown in Fig. 6 (b). The peak appeared at $480 \mathrm{~cm}^{-1}$ is known to correspond to amorphous structure of $\mathrm{Si}(\mathrm{a}-\mathrm{Si})$. The peak at $525 \mathrm{~cm}^{-1}$ in (b) has a broader width compared with that in (a), indicating poly-Si (p-Si) formation [16]. The same Raman spectra as Fig. 6 (b) were observed at the whitish part in cross-sectional SEM images shown in Fig. 5. These Raman observations indicate that the laser-induced change in $\mathrm{Si}$ corresponds to structure change of $\mathrm{c}-\mathrm{Si}$ to $\mathrm{p}-\mathrm{Si}$ and a-Si. These structural changes accompany with the changes of the refractive index, which should appear as gray lines in IR images. Gray lines observed in Fig. 2 suggest that similar structural change would occur inside Si substrate even though no change was appeared on both surfaces.

\subsection{Laser assisted backside etching}

Irradiation of the focused infrared femtosecond laser pulses at the back surface of Si substrate through itself could not machine a groove on the back surface. Even with compensation of aberration effect, only a local structure change inside and at the surface of Si substrates can be induced. The structure change induced in $\mathrm{Si}$ resulted in change of the etch rate by hot $\mathrm{KOH}$ solution. Therefore, wet etching with $\mathrm{KOH}$ solution was tried to form a groove on the back surface of Si.
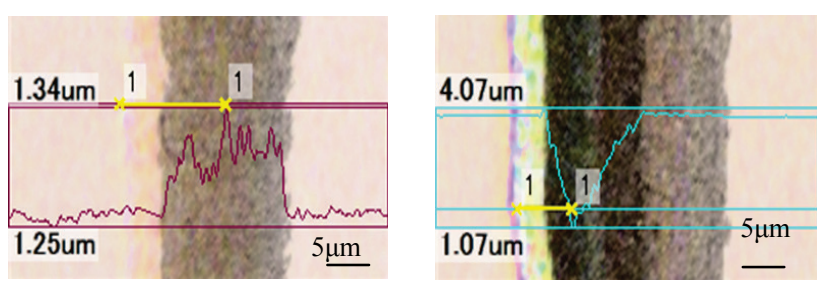

Fig.7 Laser-micrograph images and profiles of lines made on the back of Si substrate. Left is focused at -15 $\mu \mathrm{m}$ and ridge of $50 \mathrm{~nm}$ high was observed. Right is focused at $-6 \mu \mathrm{m}$ and groove of $3 \mu \mathrm{m}$ depth was observed.

A Si substrate was put on a metal cell filled with $\mathrm{KOH}$ solution. Si substrates were used as windows through which the laser was irradiated. The laser was focused on the back surface of the Si window and the cell was moved relative to the laser beam to machine a line groove.

Figure 7 shows laser micrograph images and profile of the machined lines. The correction collar position during machining was set at 0.600 . Left of Fig. 7 is obtained for the focus on $-15 \mu \mathrm{m}$; a small ridge of $50 \mathrm{~nm}$ high and 17 $\mu \mathrm{m}$ wide is observed. Right is obtained for the focus on -6 $\mu \mathrm{m}$ and a groove of $3 \mu \mathrm{m}$ deep and $13 \mu \mathrm{m}$ wide is observed. Grooves on the back surface of few micrometers were machined for focus position close to the back surface but slightly penetrated into $\mathrm{KOH}$ solution. These results suggest that wet etching of $\mathrm{Si}$ on the backside would be possible by irradiation through $\mathrm{Si}$ itself. Our results were, however, not stable; possibly due to formation bubbles at irradiated position. Further study on backside wet etching is in progress.

\section{Conclusion}

Machining of Si was performed using a femtosecond laser at $1552.5 \mathrm{~nm}$ with a machining system that employed the IR microscope and objective lens equipped with a correction collar.

To observe the back surface of $320 \mu \mathrm{m}$ thick Si through IR microscope, it was found that the correction collar should be set at 0.575 in our experimental set-up. To focus the laser light at $1552 \mathrm{~nm}$ on the back surface, additional correction was required and the collar needed to be set at 0.600 .

Black lines consisted from fine particle-like structure was observed on the back surface of the Si under that condition. IR microscope images and cross-sectional views indicate that the change extends from rear surface towered inside and the changes are sometimes observed only inside. The front surface corresponds to these lines showed no damage. The Raman observations indicate that the laser-induced change in Si corresponds to structure change of c-Si to $\mathrm{p}-\mathrm{Si}$ and $\mathrm{a}-\mathrm{Si}$.

Grooves of a few micrometers deep were machined on the back surface of Si substrate by laser irradiation through it using $\mathrm{KOH}$ solution as an etchant.

We have demonstrated that selective modification and machining of inside and back surface of Si substrate by irradiating the non-absorbed infrared laser through it is achieved. 


\section{Acknowledgments}

The authors would like to thank Mr. Tada and Mr. Sakurai (Citizen Finetech Miyota Co.) for providing the Si samples, support in experiment and helpful discussion. We also thank to reviewers for their helpful suggestions in improving the manuscript.

\section{References}

[1] Y. Shimotsuma, K. Hirao, P. G. Kazanski and J. Qiu: Japanese Journal of Applied Physics, 44, No. 7A, (2005) 4735.

[2] R. R. Gattass, E. Mazur: Nature Photonics, 2, (2008) 219.

[3] K. Atsumi: The Japan Society of Mechanical Engineers, No. 1068, 110, (2007) 14 (in Japanese)

[4] F. Fukuyo, K. Fukumitsu and N. Uchiyama: Proceedings of LPM 2005 (2005)

[5] K. Fukumitsu, M. Kumagai, E. Ohmura, H. Morita, K. Atsumi and N. Uchiyama: Proceedings of LAMP2006 (2006)

[6] E. Ohmura, F. Fukuyo, K. Fukumitsu, H. Morita: J. Achievements in Materials and Manufacturing Engineering, 17 (1-2), (2006) 381

[7] T E. Ohmura, M. Kumagai, K. Fukumitsu, M. Nakano,
N. Uchiyama and H. Morita: Journal of the Japan Society for Precision Engineering, 74 (3), (2008) 275, (in Japanese).

[8] Chen, X. Hou, S. Kanehira, K. Miura and K. Hirao: Appl. Phys. Lett., 93, (2008) 051112.

[9] C. Li, T. Chen, J. Si, F. Chen, X. Shi and X. Hou: J. Micromech. Microeng., 19, (2009) 125007.

[10] C. Li, X. Shi, J. Si, F. Chen, T. Chen Y. Zhang, X. Hou: Appl. Phys. B, 98 (2010) 377.

[11]A. H. Nejadmalayeri, P. Herman, J. Burghoff, M. Will, S. Norte and A. Tunnermann: Optics Letters, 30 (9), (2005) 964.

[12]Rie Tanabe, Fumiya Sato, Yuki Shinohe, Kozo Tada and Yoshiro Ito: Proceedings of LPM2010, (2010).

[13] Y. Ito, F. Sato, Y. Shinohe, R. Tanabe, K. Tada: Proc. SPIE 7584, (2010) 75840M

[14] Y. Ito, R. Tanabe and K. Tada: MRS Symposium Proceedings 1365, (2011) 31

[15] Y. Ito, Y. Morita, H. Sakashita, R. Suzuki, R. Tanabe, H. Sakurai, K. Tada: Proceedings of $31^{\text {st }}$ ICALEO, 105, (2012) 1102

[16] V. V. P. Sreenivas, M. Bulters and R. B. Bergmann: J. Europ. Opt. Soc. Rap. Public., 7, (2012) 12035

[17] J. Yan: Journal of Applied Physics 95 (4), (2004) 2094

(Received: August 30, 2013, Accepted: March 17, 2014) 